\title{
Predicting the Energy of the Water Exchange Reaction and Free Energy of Solvation for the Uranyl Ion in Aqueous Solution
}

Keith E. Gutowski, and David. A Dixon*,1

Department of Chemistry and Center for Green Manufacturing, The University of Alabama, Shelby Hall, Box 870336, Tuscaloosa, AL 35487, USA.

\section{Supplementary Material}

\section{Effect of varying the atomic radii on the PCM approach}

Explicit radii sets were used to examine the effects of small, incremental changes in radii on the reaction energetics and these results are included as Supplementary Information. The choice of uranium radius is relatively inconsequential, likely because it is buried within the core and effects the size very little, resulting in a variation in the corrected solvation free energy of only $2.2 \mathrm{kcal} / \mathrm{mol}$ over a $1 \AA$ range. The effect becomes larger as the uranium radius approaches $2 \AA$. Over the 1.5-2.0 interval, the change in $\Delta \mathrm{G}_{\text {corr }}$ provides results which are consistent with the use of a small solute cavity; the fact that the volume of $\mathrm{UO}_{2}\left(\mathrm{H}_{2} \mathrm{O}\right)_{4}{ }^{2+}$ actually gets smaller (Table SM2) shows that there are complicating features in terms of the interplay between the various radii. The sensitivity due to changes in the oxygen radius is much greater. An increase in the radius by $0.2 \AA$ (sets $3-5$ ) results in an increase in the corrected solvation free energy by $\sim 5 \mathrm{kcal} / \mathrm{mol}$ (Table SM3), indicative of a larger cavity, as indicated by the volumes in Table SM2. The hydrogen radius is an even more sensitive parameter. An increase of $0.1 \AA$ (sets 4 and 6) resulted in an increase in the solvation energy by $\sim 3$ $\mathrm{kcal} / \mathrm{mol}$. Larger hydrogen radii were not tested, as this would yield a cavity that is too large.)

\footnotetext{
${ }^{1}$ Email: dadixon@ama.ua.edu
} 
Table SM1. Explicit radii sets, in $\AA$. All scaling factors were set to 1.0.

$\mathrm{U} \quad \mathrm{O} \quad \mathrm{H}$

\begin{tabular}{llll} 
Set 1 & 1.0 & 1.4 & 1.2 \\
Set 2 & 1.5 & 1.4 & 1.2 \\
Set 3 & 2.0 & 1.4 & 1.2 \\
Set 4 & 2.0 & 1.5 & 1.2 \\
Set 5 & 2.0 & 1.6 & 1.2 \\
Set 6 & 2.0 & 1.5 & 1.3 \\
\hline
\end{tabular}

Table SM2. Volumes $\left(\AA^{3}\right)$ and surface areas $\left(\AA^{2}\right)$ of $\mathrm{UO}_{2}\left(\mathrm{H}_{2} \mathrm{O}\right)_{4}{ }^{2+}, \mathrm{UO}_{2}\left(\mathrm{H}_{2} \mathrm{O}\right)_{5}{ }^{2+}$, and $\mathrm{H}_{2} \mathrm{O}$ cavities from explicit radii sets 1-6.

\begin{tabular}{|l|l|l|l|l|l|l|}
\hline & $\mathbf{U O}_{2}\left(\mathbf{H}_{2} \mathbf{O}_{4}{ }^{2}\right.$ & & $\mathbf{U O}_{2}\left(\mathbf{H}_{2} \mathbf{O}\right)_{5}{ }^{2}$ & & $\mathbf{H}_{\mathbf{2}} \mathbf{O}$ & \\
\hline Cavity & Volume & SA & Volume & SA & Volume & SA \\
\hline Set 1 & 120 & 155 & 145 & 171 & 18 & 34 \\
\hline Set 2 & 120 & 154 & 145 & 171 & 18 & 34 \\
\hline Set 3 & 116 & 149 & 145 & 171 & 18 & 34 \\
\hline Set 4 & 124 & 154 & 150 & 173 & 19 & 36 \\
\hline Set 5 & 135 & 160 & 158 & 174 & 21 & 38 \\
\hline Set 6 & 137 & 165 & 164 & 182 & 21 & 39 \\
\hline
\end{tabular}


Table SM3. Electrostatic and nonelectrostatic solvation contributions, and exhange and standard-state corrected free energies (kcal/mol) for reaction (1) using Sets 1-6.

\begin{tabular}{|l|l|l|l|c|c|c|}
\hline & $\begin{array}{l}\text { Electro- } \\
\text { static }\end{array}$ & $\begin{array}{l}\text { Nonelec- } \\
\text { trostatic }\end{array}$ & Sum & $\Delta \mathbf{G}_{\text {exchange }}$ & $\Delta \mathbf{G}_{\text {corr }}$ & $\boldsymbol{\Delta}_{\text {theory-exp }}{ }^{\mathbf{2}}$ \\
\hline Set 1 & 22.0 & 2.6 & 24.6 & 7.1 & 2.8 & +4.0 \\
\hline Set 2 & 21.6 & 2.4 & 24.0 & 6.5 & 2.2 & +3.4 \\
\hline Set 3 & 23.9 & 1.8 & 25.7 & 8.7 & 4.4 & +5.6 \\
\hline Set 4 & 22.6 & 1.5 & 24.1 & 6.6 & 2.3 & +3.5 \\
\hline Set 5 & 20.3 & 0.5 & 20.8 & 3.3 & -1.0 & +0.2 \\
\hline Set 6 & 19.8 & 1.6 & 21.4 & 3.9 & -0.4 & +0.8 \\
\hline
\end{tabular}


Molecular $x, y, z$ coordinates of the optimized structures in $\AA$.

\section{Optimized Coordinates}

\section{$\mathrm{H}_{2} \mathrm{O} \mathrm{C}_{2 \mathrm{v}}$ (B3LYP)}

Standard orientation:

\begin{tabular}{|c|c|c|c|c|}
\hline \multirow{2}{*}{$\begin{array}{l}\text { Center } \\
\text { Number }\end{array}$} & \multirow{2}{*}{$\begin{array}{l}\text { Atomic } \\
\text { Number }\end{array}$} & \multirow{2}{*}{$\begin{array}{l}\text { Atomic } \\
\text { Type }\end{array}$} & \multicolumn{2}{|c|}{ Coordinates (Angstroms } \\
\hline & & & X $\quad Y$ & $\mathrm{Z}$ \\
\hline 1 & 8 & 0.000000 & 0.000000 & 0.116775 \\
\hline 2 & 1 & 0.000000 & 0.767139 & -0.467101 \\
\hline 3 & 1 & 0.000000 & -0.767139 & -0.467101 \\
\hline
\end{tabular}

$\left(\mathrm{H}_{2} \mathrm{O}\right)_{2} \mathrm{C}_{1}$ (B3LYP)

Standard orientation:

\begin{tabular}{lllrrr} 
Center & \multicolumn{2}{c}{ Atomic } & \multicolumn{2}{c}{ Atomic } & \multicolumn{2}{c}{ Coordinates (Angstrom } \\
Number & Number & Type & X & Y & Z \\
- \hdashline 1 & 8 & 0 & -1.379348 & -0.013108 & 0.103747 \\
2 & 1 & 0 & -1.742518 & 0.815235 & -0.232866 \\
3 & 1 & 0 & -1.800546 & -0.713100 & -0.409922 \\
4 & 1 & 0 & 0.552593 & -0.026031 & -0.001170 \\
5 & 8 & 0 & 1.516986 & 0.016117 & -0.118517 \\
6 & 1 & 0 & 1.889371 & -0.100174 & 0.762120
\end{tabular}

$\left(\mathrm{H}_{2} \mathrm{O}\right)_{3} \mathrm{C}_{1}$ (B3LYP)

Standard orientation:

\begin{tabular}{|c|c|c|c|c|}
\hline \multirow{2}{*}{$\begin{array}{l}\text { Center } \\
\text { Number }\end{array}$} & \multirow{2}{*}{$\begin{array}{l}\text { Atomic } \\
\text { Number }\end{array}$} & \multirow{2}{*}{$\begin{array}{l}\text { Atomic } \\
\text { Type }\end{array}$} & \multicolumn{2}{|c|}{ Coordinates (Angstrom } \\
\hline & & & $\mathrm{X}$ & $\mathrm{Z}$ \\
\hline 1 & 8 & -1.307269 & -0.931477 & -0.093280 \\
\hline 2 & 8 & -0.159010 & 1.591371 & 0.110542 \\
\hline 3 & 8 & 1.466709 & -0.651404 & -0.083126 \\
\hline 4 & 1 & 2.008915 & -1.087584 & 0.583266 \\
\hline 5 & 1 & 0.590900 & -1.088249 & -0.064322 \\
\hline 6 & 1 & -1.950398 & -1.191813 & 0.575158 \\
\hline 7 & 1 & -1.214061 & 0.041732 & -0.028201 \\
\hline 8 & 1 & -0.081373 & 2.228419 & -0.608633 \\
\hline 9 & 1 & 0.642578 & 1.029575 & 0.069646 \\
\hline
\end{tabular}

$\left(\mathrm{H}_{2} \mathrm{O}\right)_{4} \mathrm{C}_{1}$ (B3LYP)

Standard orientation: 


\begin{tabular}{|c|c|c|c|c|c|}
\hline Center & Atomic & & omic & Coordinate & $(\mathrm{A}$ \\
\hline Number & Numbe & & Туре & X & Z \\
\hline 1 & 8 & 0 & 0.568730 & -1.839850 & -0.003689 \\
\hline 2 & 1 & 0 & -0.361504 & -1.506112 & -0.018979 \\
\hline 3 & 1 & 0 & 0.625568 & -2.455519 & 0.735555 \\
\hline 4 & 1 & 0 & 1.506114 & -0.361506 & 0.018964 \\
\hline 5 & 8 & 0 & 1.839848 & 0.568729 & 0.003685 \\
\hline 6 & 1 & 0 & 2.455533 & 0.625575 & -0.735546 \\
\hline 7 & 8 & 0 & -1.839848 & -0.568730 & 0.003685 \\
\hline 8 & 1 & 0 & -2.455538 & -0.625574 & -0.735541 \\
\hline 9 & 1 & 0 & -1.506115 & 0.361507 & 0.018965 \\
\hline 10 & 8 & 0 & -0.568730 & 1.839849 & -0.003682 \\
\hline 11 & 1 & 0 & -0.625571 & 2.455523 & 0.735557 \\
\hline 12 & 1 & 0 & 0.361505 & 1.506113 & -0.018969 \\
\hline
\end{tabular}

\section{$\left(\mathrm{H}_{2} \mathrm{O}\right)_{5} \mathrm{C}_{1}$ (B3LYP)}

Standard orientation:

\begin{tabular}{|c|c|c|c|c|c|}
\hline \multirow{2}{*}{$\begin{array}{l}\text { Center } \\
\text { Number }\end{array}$} & \multirow{2}{*}{$\begin{array}{l}\text { Atomic } \\
\text { Number }\end{array}$} & \multirow{2}{*}{\multicolumn{2}{|c|}{$\begin{array}{l}\text { Atomic } \\
\text { Type }\end{array}$}} & \multicolumn{2}{|c|}{ Coordinates (Angstroms } \\
\hline & & & & $\mathrm{X}$ & $\mathrm{Z}$ \\
\hline 1 & 8 & 0 & 2.291262 & -0.242646 & -0.049233 \\
\hline 2 & 8 & 0 & 0.476300 & -2.248128 & -0.051649 \\
\hline 3 & 8 & 0 & -1.989859 & -1.151651 & 0.121348 \\
\hline 4 & 8 & 0 & -1.716081 & 1.529519 & -0.093543 \\
\hline 5 & 8 & 0 & 0.924763 & 2.114740 & 0.013787 \\
\hline 6 & 1 & 0 & 2.990098 & -0.476307 & 0.570961 \\
\hline 7 & 1 & 0 & 0.445438 & -2.817055 & -0.828969 \\
\hline 8 & 1 & 0 & -2.540553 & -1.328170 & 0.892039 \\
\hline 9 & 1 & 0 & -2.041552 & 1.962909 & -0.890395 \\
\hline 10 & 1 & 0 & 1.257122 & 2.628715 & 0.757674 \\
\hline 11 & 1 & 0 & 1.657875 & -1.004975 & -0.055910 \\
\hline 12 & 1 & 0 & -0.436165 & -1.874295 & 0.047286 \\
\hline 13 & 1 & 0 & -1.940493 & -0.165738 & 0.033632 \\
\hline 14 & 1 & 0 & -0.756704 & 1.767119 & -0.025995 \\
\hline 15 & 1 & 0 & 1.473858 & 1.293123 & -0.025999 \\
\hline
\end{tabular}

\section{$\left(\mathrm{H}_{2} \mathrm{O}\right)_{12} \mathrm{C}_{1}$ (B3LYP)}

Standard orientation:

\begin{tabular}{|c|c|c|c|c|}
\hline Center & Atomic & Atomic & Coordinate & es (Angstror \\
\hline Number & Number & Type & $\mathrm{X}$ & Z \\
\hline 1 & 8 & 2.868276 & 1.929706 & 0.533467 \\
\hline 2 & 8 & 2.815496 & 0.637365 & -1.811904 \\
\hline
\end{tabular}




$\begin{array}{cccccc}3 & 8 & 0 & 0.040596 & 1.836141 & 0.655772 \\ 4 & 8 & 0 & -0.040692 & 0.655694 & -1.834282 \\ 5 & 8 & 0 & 0.040793 & -1.834367 & -0.654071 \\ 6 & 8 & 0 & -0.040429 & -0.654359 & 1.836048 \\ 7 & 8 & 0 & -2.868901 & -0.534556 & 1.929242 \\ 8 & 8 & 0 & -2.816215 & 1.811151 & 0.636928 \\ 9 & 8 & 0 & -2.868208 & 0.533345 & -1.930028 \\ 10 & 8 & 0 & -2.815485 & -1.811955 & -0.638008 \\ 11 & 8 & 0 & 2.868620 & -1.930318 & -0.534352 \\ 12 & 8 & 0 & 2.816046 & -0.637701 & 1.811059 \\ 13 & 1 & 0 & 0.043468 & 0.999205 & 1.179734 \\ 14 & 1 & 0 & 0.951225 & 2.182214 & 0.723602 \\ 15 & 1 & 0 & 1.900609 & 0.695897 & -2.143649 \\ 16 & 1 & 0 & 2.940455 & -0.298870 & -1.560343 \\ 17 & 1 & 0 & 0.045115 & -0.997151 & -1.177635 \\ 18 & 1 & 0 & 0.950585 & -2.182391 & -0.722846 \\ 19 & 1 & 0 & 2.939582 & 0.298656 & 1.559184 \\ 20 & 1 & 0 & 1.901801 & -0.697052 & 2.144368 \\ 21 & 1 & 0 & 2.918511 & 1.528342 & -0.379551 \\ 22 & 1 & 0 & 3.555256 & 2.604276 & 0.585262 \\ 23 & 1 & 0 & -0.044319 & 1.179415 & -0.997152 \\ 24 & 1 & 0 & -0.044296 & -1.177781 & 0.998739 \\ 25 & 1 & 0 & 2.918293 & -1.528999 & 0.378697 \\ 26 & 1 & 0 & 3.555422 & -2.605115 & -0.585555 \\ 27 & 1 & 0 & -1.900748 & -2.144038 & -0.696897 \\ 28 & 1 & 0 & -2.939629 & -1.559589 & 0.298159 \\ 29 & 1 & 0 & -2.917899 & -0.379974 & -1.529235 \\ 30 & 1 & 0 & -2.939289 & 1.558794 & -0.299372 \\ 31 & 1 & 0 & -1.902315 & 2.145312 & 0.696403 \\ 32 & 1 & 0 & -2.917346 & 0.378888 & 1.528707 \\ 33 & 1 & 0 & -0.950161 & -0.723876 & 2.183957 \\ 34 & 1 & 0 & -0.950977 & 0.723660 & -2.181225 \\ 35 & 1 & 0 & -3.557365 & -0.586141 & 2.602316 \\ 36 & 1 & 0 & -3.555151 & 0.585146 & -2.604633 \\ -------------------------------------------------------------\end{array}$

\section{$\left(\mathrm{H}_{2} \mathrm{O}\right)_{15} \mathrm{C}_{1}$ (B3LYP)}

Standard orientation:

\begin{tabular}{|c|c|c|c|c|}
\hline Center & Atomic & Atomic & \multicolumn{2}{|c|}{ Coordinates (Angstron } \\
\hline Number & Numbe & Type & $X$ & $\mathrm{Z}$ \\
\hline 1 & 8 & 3.061472 & -0.340436 & 2.209655 \\
\hline 2 & 1 & 3.139716 & -1.057017 & 1.533236 \\
\hline 3 & 1 & 3.769701 & -0.479194 & 2.849321 \\
\hline 4 & 8 & 3.006961 & -2.228492 & 0.212235 \\
\hline 5 & 1 & 2.938663 & -1.768397 & -0.679915 \\
\hline 6 & 1 & 3.654337 & -2.935680 & 0.105910 \\
\hline 7 & 8 & 2.682709 & -1.009846 & -2.108157 \\
\hline
\end{tabular}




$\begin{array}{cccccc}8 & 1 & 0 & 2.804189 & -0.038098 & -2.060590 \\ 9 & 1 & 0 & 1.751335 & -1.142077 & -2.367512 \\ 10 & 8 & 0 & 2.760650 & 1.792861 & -1.754369 \\ 11 & 1 & 0 & 3.388906 & 2.381222 & -2.189218 \\ 12 & 1 & 0 & 2.830599 & 1.967039 & -0.772138 \\ 13 & 8 & 0 & 2.736578 & 2.188132 & 0.884625 \\ 14 & 1 & 0 & 1.809287 & 2.399189 & 1.104303 \\ 15 & 1 & 0 & 2.937951 & 1.369735 & 1.379476 \\ 16 & 8 & 0 & -0.104059 & 2.063072 & 1.012320 \\ 17 & 1 & 0 & -0.122744 & 1.895579 & 0.036199 \\ 18 & 1 & 0 & -1.017561 & 2.332754 & 1.235148 \\ 19 & 1 & 0 & 0.087567 & 0.560894 & 1.854737 \\ 20 & 8 & 0 & 0.170228 & -0.309921 & 2.319837 \\ 21 & 1 & 0 & 1.092602 & -0.345210 & 2.628664 \\ 22 & 8 & 0 & -0.078803 & 1.578419 & -1.671020 \\ 23 & 1 & 0 & 0.812501 & 1.868489 & -1.941764 \\ 24 & 1 & 0 & -0.083287 & 0.596663 & -1.802855 \\ 25 & 8 & 0 & -0.162353 & -1.122501 & -1.996981 \\ 26 & 1 & 0 & -0.101903 & -1.561382 & -1.111187 \\ 27 & 1 & 0 & -1.087582 & -1.259836 & -2.283365 \\ 28 & 8 & 0 & 0.113470 & -2.299954 & 0.450142 \\ 29 & 1 & 0 & 1.036605 & -2.609570 & 0.435527 \\ 30 & 1 & 0 & 0.099387 & -1.587705 & 1.138282 \\ 31 & 8 & 0 & -2.918434 & 2.097324 & 1.146486 \\ 32 & 1 & 0 & -3.018281 & 1.915419 & 0.168324 \\ 33 & 1 & 0 & -3.587836 & 2.752018 & 1.377028 \\ 34 & 8 & 0 & -2.951533 & 1.612869 & -1.478501 \\ 35 & 1 & 0 & -3.076482 & 0.666842 & -1.704206 \\ 36 & 1 & 0 & -2.048251 & 1.824996 & -1.774881 \\ 37 & 8 & 0 & -2.983703 & -1.176882 & -1.979424 \\ 38 & 1 & 0 & -2.993787 & -1.630890 & -1.081483 \\ 39 & 1 & 0 & -3.657066 & -1.609704 & -2.517232 \\ 40 & 8 & 0 & -2.805268 & -2.359244 & 0.371564 \\ 41 & 1 & 0 & -1.894193 & -2.692243 & 0.436845 \\ 42 & 1 & 0 & -2.891968 & -1.724958 & 1.125093 \\ 43 & 8 & 0 & -2.756993 & -0.486895 & 2.398199 \\ 44 & 1 & 0 & -1.832845 & -0.489299 & 2.699278 \\ 45 & 1 & 0 & -2.906932 & 0.412378 & 2.046082 \\ ---------------------------------------------------------\end{array}$

$\mathrm{UO}_{2}\left(\mathrm{H}_{2} \mathrm{O}\right)_{4}{ }^{{ }^{+}} \mathrm{D}_{4 \mathrm{~h}}(\mathrm{~B} 3 \mathrm{LYP})$

Standard orientation:

\begin{tabular}{|c|c|c|c|}
\hline Center & Atomic & Atomic & Coordinates (Angstron \\
\hline Number & Number & Type & $\mathrm{X}$ \\
\hline 1 & 92 & 0.000000 & $0.000000 \quad 0.000000$ \\
\hline 2 & 8 & 0.000000 & $0.000000 \quad 1.745614$ \\
\hline 3 & 8 & 0.000000 & $0.000000-1.745614$ \\
\hline
\end{tabular}




$\begin{array}{cccccc}4 & 8 & 0 & 0.000000 & 2.435760 & -0.000000 \\ 5 & 1 & 0 & 0.000000 & 3.015279 & 0.782516 \\ 6 & 1 & 0 & 0.000000 & 3.015279 & -0.782516 \\ 7 & 1 & 0 & 3.015279 & -0.000000 & -0.782516 \\ 8 & 8 & 0 & 2.435760 & -0.000000 & -0.000000 \\ 9 & 1 & 0 & 3.015279 & -0.000000 & 0.782516 \\ 10 & 8 & 0 & -0.000000 & -2.435760 & -0.000000 \\ 11 & 1 & 0 & -0.000000 & -3.015279 & -0.782516 \\ 12 & 1 & 0 & -0.000000 & -3.015279 & 0.782516 \\ 13 & 1 & 0 & -3.015279 & 0.000000 & 0.782516 \\ 14 & 8 & 0 & -2.435760 & 0.000000 & -0.000000 \\ 15 & 1 & 0 & -3.015279 & 0.000000 & -0.782516 \\ --------------------------------------------------------------------\end{array}$

\section{$\mathrm{UO}_{2}\left(\mathrm{H}_{2} \mathrm{O}\right)_{4}{ }^{2+} \mathrm{D}_{4}(\mathrm{SVWN})$}

Standard orientation:

\begin{tabular}{cccccc} 
Center & \multicolumn{2}{c}{ Atomic } & \multicolumn{2}{c}{ Atomic } & \multicolumn{3}{c}{ Coordinates } & Angstroms) \\
Number & Number & Type & X & Y & $Z$ \\
-------------- & Z \\
1 & 92 & 0 & 0.000000 & 0.000000 & 0.000000 \\
2 & 8 & 0 & 0.000000 & 0.000000 & 1.754502 \\
3 & 8 & 0 & 0.000000 & 0.000000 & -1.754502 \\
4 & 8 & 0 & 0.000000 & 2.354746 & 0.000000 \\
5 & 1 & 0 & -0.000202 & 2.936878 & 0.794746 \\
6 & 1 & 0 & 0.000202 & 2.936878 & -0.794746 \\
7 & 1 & 0 & 2.936878 & -0.000202 & -0.794746 \\
8 & 8 & 0 & 2.354746 & 0.000000 & 0.000000 \\
9 & 1 & 0 & 2.936878 & 0.000202 & 0.794746 \\
10 & 8 & 0 & 0.000000 & -2.354746 & 0.000000 \\
11 & 1 & 0 & -0.000202 & -2.936878 & -0.794746 \\
12 & 1 & 0 & 0.000202 & -2.936878 & 0.794746 \\
13 & 1 & 0 & -2.936878 & -0.000202 & 0.794746 \\
14 & 8 & 0 & -2.354746 & 0.000000 & 0.000000 \\
15 & 1 & 0 & -2.936878 & 0.000202 & -0.794746 \\
---------------------------------------------------------
\end{tabular}

\section{$\mathrm{UO}_{2}\left(\mathrm{H}_{2} \mathrm{O}\right)_{5}{ }^{2+} \mathrm{D}_{5 \mathrm{~h}}(\mathrm{~B} 3 \mathrm{LYP})$}

Standard orientation:

\begin{tabular}{lccccc} 
Center & \multicolumn{2}{c}{ Atomic } & \multicolumn{2}{c}{ Atomic } & \multicolumn{2}{c}{ Coordinates (Angstroms) } \\
Number & Number & Type & X & Y & Z \\
\hline 1 & 92 & 0 & 0.000000 & 0.000000 & 0.000000 \\
2 & 8 & 0 & 0.000000 & 0.000000 & 1.748343 \\
3 & 8 & 0 & 0.000000 & 0.000000 & -1.748343 \\
4 & 8 & 0 & 0.000000 & 2.499560 & 0.000000 \\
5 & 1 & 0 & 0.000000 & 3.077065 & 0.780560 \\
6 & 1 & 0 & 0.000000 & 3.077065 & -0.780560
\end{tabular}




$\begin{array}{cccccc}7 & 1 & 0 & 2.926463 & 0.950865 & -0.780560 \\ 8 & 8 & 0 & 2.377223 & 0.772407 & 0.000000 \\ 9 & 1 & 0 & 2.926463 & 0.950865 & 0.780560 \\ 10 & 8 & 0 & -1.469205 & -2.022187 & 0.000000 \\ 11 & 1 & 0 & -1.808653 & -2.489398 & -0.780560 \\ 12 & 1 & 0 & -1.808653 & -2.489398 & 0.780560 \\ 13 & 1 & 0 & -2.926463 & 0.950865 & 0.780560 \\ 14 & 8 & 0 & -2.377223 & 0.772407 & 0.000000 \\ 15 & 1 & 0 & -2.926463 & 0.950865 & -0.780560 \\ 16 & 1 & 0 & 1.808653 & -2.489398 & -0.780560 \\ 17 & 8 & 0 & 1.469205 & -2.022187 & 0.000000 \\ 18 & 1 & 0 & 1.808653 & -2.489398 & 0.780560 \\ ------------------------------------------------------------------\end{array}$

\section{$\mathrm{UO}_{2}\left(\mathrm{H}_{2} \mathrm{O}\right)_{5}{ }^{2+} \mathrm{D}_{5}$ (B3LYP)}

Standard orientation:

\begin{tabular}{cccccc} 
Center & \multicolumn{2}{c}{ Atomic } & \multicolumn{2}{c}{ Atomic } & \multicolumn{3}{c}{ Coordinates } \\
Number & Number & Type & X & Y & $Z$ \\
------- & Z \\
\hline 1 & 92 & 0 & 0.000000 & 0.000000 & 0.000000 \\
2 & 8 & 0 & 0.000000 & 0.000000 & 1.748343 \\
3 & 8 & 0 & 0.000000 & -0.000000 & -1.748343 \\
4 & 8 & 0 & -0.000000 & 2.499560 & 0.000000 \\
5 & 1 & 0 & 0.254057 & 3.077065 & 0.738058 \\
6 & 1 & 0 & -0.254057 & 3.077065 & -0.738058 \\
7 & 1 & 0 & 2.847955 & 1.192488 & -0.738058 \\
8 & 8 & 0 & 2.377223 & 0.772407 & 0.000000 \\
9 & 1 & 0 & 3.004971 & 0.709243 & 0.738058 \\
10 & 8 & 0 & -1.469205 & -2.022187 & 0.000000 \\
11 & 1 & 0 & -1.603118 & -2.638729 & -0.738058 \\
12 & 1 & 0 & -2.014190 & -2.340068 & 0.738058 \\
13 & 1 & 0 & -2.847955 & 1.192488 & 0.738058 \\
14 & 8 & 0 & -2.377223 & 0.772407 & 0.000000 \\
15 & 1 & 0 & -3.004971 & 0.709243 & -0.738058 \\
16 & 1 & 0 & 2.014190 & -2.340068 & -0.738058 \\
17 & 8 & 0 & 1.469205 & -2.022187 & 0.000000 \\
18 & 1 & 0 & 1.603118 & -2.638729 & 0.738058 \\
---------------------------------------------------------
\end{tabular}

\section{$\mathrm{UO}_{2}\left(\mathrm{H}_{2} \mathrm{O}\right)_{5}{ }^{2+} \mathrm{C}_{1}$ (B3LYP)}

Standard orientation:

\begin{tabular}{lccccc} 
Center & Atomic & \multicolumn{2}{c}{ Atomic } & \multicolumn{2}{c}{ Coordinates (Angstrom } \\
Number & Number & Type & X & Y & Z \\
-1 & 92 & 0 & 0.000000 & 0.000000 & 0.000000 \\
2 & 8 & 0 & 0.000000 & 0.000000 & -1.748343 \\
3 & 8 & 0 & 0.000000 & 0.000000 & 1.748343
\end{tabular}




$\begin{array}{cccccc}4 & 8 & 0 & 2.230374 & 1.128376 & 0.000000 \\ 5 & 1 & 0 & 2.619408 & 1.638682 & -0.728716 \\ 6 & 1 & 0 & 2.871963 & 1.139476 & 0.728716 \\ 7 & 1 & 0 & -0.183697 & 3.085464 & 0.723723 \\ 8 & 8 & 0 & -0.383926 & 2.469899 & 0.000000 \\ 9 & 1 & 0 & -0.761562 & 2.995639 & -0.723723 \\ 10 & 8 & 0 & -1.141168 & -2.223857 & 0.000000 \\ 11 & 1 & 0 & -1.630921 & -2.621642 & 0.738034 \\ 12 & 1 & 0 & -1.178731 & -2.853683 & -0.738034 \\ 13 & 1 & 0 & 2.358873 & -1.993821 & -0.733487 \\ 14 & 8 & 0 & 1.762373 & -1.772524 & 0.000000 \\ 15 & 1 & 0 & 1.980241 & -2.370285 & 0.733487 \\ 16 & 1 & 0 & -2.999370 & 0.728212 & 0.742357 \\ 17 & 8 & 0 & -2.467654 & 0.398106 & 0.000000 \\ 18 & 1 & 0 & -3.076204 & 0.251958 & -0.742357\end{array}$

\section{$\mathrm{UO}_{2}\left(\mathrm{H}_{2} \mathrm{O}\right)_{5}{ }^{2+} \mathrm{D}_{5}(\mathrm{SVWN})$}

Standard orientation:

\begin{tabular}{|c|c|c|c|c|c|}
\hline \multirow{2}{*}{$\begin{array}{l}\text { Center } \\
\text { Number }\end{array}$} & \multirow{2}{*}{\multicolumn{2}{|c|}{$\begin{array}{l}\text { Atomic } \\
\text { Number }\end{array}$}} & \multirow{2}{*}{$\begin{array}{l}\text { Atomic } \\
\text { Type }\end{array}$} & \multicolumn{2}{|c|}{ Coordinates (Angstrom } \\
\hline & & & & $\mathrm{X}$ & $\mathrm{Z}$ \\
\hline 1 & 92 & 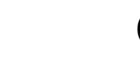 & 0.000000 & 0.000000 & 0.000000 \\
\hline 2 & 8 & 0 & 0.000000 & 0.000000 & 1.757826 \\
\hline 3 & 8 & 0 & 0.000000 & 0.000000 & -1.757826 \\
\hline 4 & 8 & 0 & 0.000000 & 2.412865 & 0.000000 \\
\hline 5 & 1 & 0 & 0.252465 & 2.993258 & 0.750999 \\
\hline 6 & 1 & 0 & -0.252465 & 2.993258 & -0.750999 \\
\hline 7 & 1 & 0 & 2.768741 & 1.165076 & -0.750999 \\
\hline 8 & 8 & 0 & 2.294771 & 0.745616 & 0.000000 \\
\hline 9 & 1 & 0 & 2.924773 & 0.684859 & 0.750999 \\
\hline 10 & 8 & 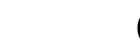 & -1.418246 & -1.952049 & 0.000000 \\
\hline 11 & 1 & & -1.555144 & -2.569992 & -0.750999 \\
\hline 12 & 1 & & -1.963642 & -2.273201 & 0.750999 \\
\hline 13 & 1 & & -2.768741 & 1.165076 & 0.750999 \\
\hline 14 & 8 & ( & -2.294771 & 0.745616 & 0.000000 \\
\hline 15 & 1 & 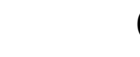 & -2.924773 & 0.684859 & -0.750999 \\
\hline 16 & 1 & 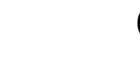 & 1.963642 & -2.273201 & -0.750999 \\
\hline 17 & 8 & & 1.418246 & -1.952049 & 0.000000 \\
\hline 18 & 1 & 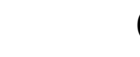 & 1.555144 & -2.569992 & 0.750999 \\
\hline
\end{tabular}

\section{$\mathrm{UO}_{2}\left(\mathrm{H}_{2} \mathrm{O}\right)_{5}{ }^{2+} \mathrm{C}_{\mathbf{1}}(\mathrm{SVWN})$}

Input orientation:

\begin{tabular}{llllc} 
Center & Atomic & Atomic & & \multicolumn{2}{c}{ Coordinates (Angstroms) } \\
Number & Number & Type & X & Y C Z
\end{tabular}




$\begin{array}{cccccc}1 & 92 & 0 & -0.000244 & 0.049749 & -0.000122 \\ 2 & 8 & 0 & 0.027660 & 0.136511 & 1.758740 \\ 3 & 8 & 0 & -0.028868 & 0.136910 & -1.758949 \\ 4 & 8 & 0 & 0.000413 & 2.439030 & 0.001236 \\ 5 & 1 & 0 & 0.009174 & 3.017098 & 0.794920 \\ 6 & 1 & 0 & -0.007737 & 3.018551 & -0.791374 \\ 7 & 1 & 0 & 2.680511 & 0.816684 & -1.234116 \\ 8 & 8 & 0 & 2.297965 & 0.645748 & -0.346102 \\ 9 & 1 & 0 & 3.010581 & 0.777678 & 0.316160 \\ 10 & 8 & 0 & -1.303053 & -1.912326 & -0.560932 \\ 11 & 1 & 0 & -1.525064 & -2.132844 & -1.491626 \\ 12 & 1 & 0 & -1.710142 & -2.596160 & 0.013812 \\ 13 & 1 & 0 & -2.682262 & 0.816546 & 1.231571 \\ 14 & 8 & 0 & -2.298519 & 0.646488 & 0.343887 \\ 15 & 1 & 0 & -3.010249 & 0.778765 & -0.319267 \\ 16 & 1 & 0 & 1.711985 & -2.594933 & -0.012414 \\ 17 & 8 & 0 & 1.303520 & -1.911511 & 0.561788 \\ 18 & 1 & 0 & 1.524328 & -2.131984 & 1.492789 \\ -------------------------------------------------------------------\end{array}$

\section{$\mathrm{UO}_{2}\left(\mathrm{H}_{2} \mathrm{O}\right)_{4}\left(\mathrm{H}_{2} \mathrm{O}\right)_{8}{ }^{2+} \mathrm{C}_{1}(\mathrm{~B} 3 \mathrm{LYP})$}

Standard orientation:

\begin{tabular}{cccccc} 
Center & \multicolumn{2}{c}{ Atomic } & \multicolumn{2}{c}{ Atomic } & \multicolumn{3}{c}{ Coordinates } \\
Number & Number & Type & X & Y & $Z$ \\
- & Numstrom & Z \\
\hline 1 & 92 & 0 & -0.004404 & -0.005884 & -0.003654 \\
2 & 8 & 0 & -0.054090 & 0.021653 & -1.767666 \\
3 & 8 & 0 & 0.046050 & -0.033113 & 1.760574 \\
4 & 8 & 0 & -1.471250 & -1.868378 & 0.002940 \\
5 & 1 & 0 & -1.941521 & -2.235727 & -0.793865 \\
6 & 1 & 0 & -1.728171 & -2.407492 & 0.799434 \\
7 & 1 & 0 & 2.388115 & -1.753641 & 0.730518 \\
8 & 8 & 0 & 1.861336 & -1.464849 & -0.063348 \\
9 & 1 & 0 & 2.261698 & -1.875651 & -0.876603 \\
10 & 8 & 0 & 1.460457 & 1.855113 & -0.012191 \\
11 & 1 & 0 & 1.795281 & 2.330779 & 0.795700 \\
12 & 1 & 0 & 1.854826 & 2.285421 & -0.818647 \\
13 & 1 & 0 & -2.289345 & 1.895307 & -0.719289 \\
14 & 8 & 0 & -1.864578 & 1.458159 & 0.067764 \\
15 & 1 & 0 & -2.341429 & 1.751931 & 0.890687 \\
16 & 1 & 0 & -3.276222 & 3.572723 & -2.062807 \\
17 & 8 & 0 & -2.998590 & 2.647959 & -2.030292 \\
18 & 1 & 0 & -3.435988 & 2.201335 & -2.766525 \\
19 & 1 & 0 & -4.098667 & 2.325603 & 2.374338 \\
20 & 8 & 0 & -3.141693 & 2.243874 & 2.270972 \\
21 & 1 & 0 & -2.741620 & 2.692172 & 3.027062 \\
22 & 1 & 0 & 2.014999 & 3.441641 & -2.877877 \\
23 & 8 & 0 & 2.512120 & 3.012871 & -2.169539
\end{tabular}




$\begin{array}{llllll}24 & 1 & 0 & 3.433844 & 3.281999 & -2.275462 \\ 25 & 1 & 0 & 2.414366 & 4.086849 & 2.256546 \\ 26 & 8 & 0 & 2.356939 & 3.128353 & 2.150479 \\ 27 & 1 & 0 & 2.901773 & 2.739321 & 2.846582 \\ 28 & 8 & 0 & 2.944517 & -2.558368 & -2.238240 \\ 29 & 1 & 0 & 3.143327 & -3.494503 & -2.369515 \\ 30 & 1 & 0 & 3.370995 & -2.080033 & -2.960724 \\ 31 & 8 & 0 & 3.283578 & -2.230536 & 2.058297 \\ 32 & 1 & 0 & 2.934605 & -2.694741 & 2.830081 \\ 33 & 1 & 0 & 4.240673 & -2.362277 & 2.065648 \\ 34 & 1 & 0 & -3.699483 & -3.066807 & -2.132525 \\ 35 & 8 & 0 & -2.767400 & -2.812517 & -2.127459 \\ 36 & 1 & 0 & -2.361471 & -3.238021 & -2.893806 \\ 37 & 8 & 0 & -2.124023 & -3.337241 & 2.128752 \\ 38 & 1 & 0 & -2.089203 & -4.302161 & 2.161848 \\ 39 & 1 & 0 & -2.693205 & -3.056569 & 2.857044\end{array}$

\section{$\mathrm{UO}_{2}\left(\mathrm{H}_{2} \mathrm{O}\right)_{4}\left(\mathrm{H}_{2} \mathrm{O}\right)_{10}{ }^{2+} \mathrm{C}_{1}(\mathrm{~B} 3 \mathrm{LYP})$}

Standard orientation:

\begin{tabular}{|c|c|c|c|c|c|}
\hline \multirow{2}{*}{$\begin{array}{l}\text { Center } \\
\text { Number }\end{array}$} & \multirow{2}{*}{$\begin{array}{l}\text { Atomic } \\
\text { Number }\end{array}$} & \multirow{2}{*}{\multicolumn{2}{|c|}{$\begin{array}{l}\text { Atomic } \\
\text { Type }\end{array}$}} & \multicolumn{2}{|c|}{ Coordinates (Angstrom } \\
\hline & & & & $\mathrm{X}$ & Z \\
\hline 1 & 92 & 0 & 0.019158 & 0.125829 & 0.244671 \\
\hline 2 & 8 & 0 & 0.009392 & 0.977731 & 1.791451 \\
\hline 3 & 8 & 0 & 0.032309 & -0.735593 & -1.302207 \\
\hline 4 & 8 & 0 & 1.589873 & 1.663803 & -0.551058 \\
\hline 5 & 1 & 0 & 1.916788 & 2.458377 & -0.051865 \\
\hline 6 & 1 & 0 & 2.162656 & 1.517793 & -1.382207 \\
\hline 7 & 1 & 0 & 2.167569 & -1.963392 & 0.210844 \\
\hline 8 & 8 & 0 & 1.814080 & -1.319298 & 0.867873 \\
\hline 9 & 1 & 0 & 2.385388 & -1.337494 & 1.684435 \\
\hline 10 & 8 & 0 & -1.558481 & -1.379316 & 1.088392 \\
\hline 11 & 1 & 0 & -2.024456 & -2.086634 & 0.517895 \\
\hline 12 & 1 & 0 & -2.028983 & -1.292856 & 1.959270 \\
\hline 13 & 1 & 0 & -2.253081 & 2.200436 & -0.099233 \\
\hline 14 & 8 & 0 & -1.787932 & 1.457493 & -0.575186 \\
\hline 15 & 1 & 0 & -2.264555 & 1.286379 & -1.420139 \\
\hline 16 & 1 & 0 & -3.739933 & 3.349978 & 1.302022 \\
\hline 17 & 8 & 0 & -3.102511 & 3.442365 & 0.581978 \\
\hline 18 & 1 & 0 & -2.844397 & 4.373068 & 0.557268 \\
\hline 19 & 1 & 0 & -2.642904 & 0.779371 & -3.720958 \\
\hline 20 & 8 & 0 & -3.191381 & 0.895120 & -2.930686 \\
\hline 21 & 1 & 0 & -3.849956 & 1.565364 & -3.167945 \\
\hline 22 & 1 & 0 & -2.455273 & -0.974302 & 4.274490 \\
\hline 23 & 8 & 0 & -2.872960 & -1.079846 & 3.409885 \\
\hline 24 & 1 & 0 & -3.753769 & -1.441085 & 3.575174 \\
\hline 25 & 1 & 0 & -3.375129 & -2.620365 & -1.063403 \\
\hline
\end{tabular}




$\begin{array}{rrrrrr}26 & 8 & 0 & -2.693169 & -3.039992 & -0.483556 \\ 27 & 1 & 0 & -2.994746 & -3.928855 & -0.260900 \\ 28 & 8 & 0 & 3.353979 & -1.387678 & 3.023674 \\ 29 & 1 & 0 & 4.255302 & -1.044659 & 3.079021 \\ 30 & 1 & 0 & 3.216502 & -1.937751 & 3.805749 \\ 31 & 8 & 0 & 2.666642 & -2.959054 & -1.202322 \\ 32 & 1 & 0 & 1.961541 & -2.982568 & -1.866893 \\ 33 & 1 & 0 & 2.912059 & -3.882806 & -1.045085 \\ 34 & 1 & 0 & 2.771122 & 4.628351 & 0.381607 \\ 35 & 8 & 0 & 2.375119 & 3.846864 & 0.788871 \\ 36 & 1 & 0 & 2.605150 & 3.879517 & 1.726288 \\ 37 & 8 & 0 & 3.000394 & 1.111633 & -2.606421 \\ 38 & 1 & 0 & 3.687755 & 0.415367 & -2.464447 \\ 39 & 1 & 0 & 3.305699 & 1.704238 & -3.303170 \\ 40 & 1 & 0 & 5.503564 & -1.159172 & -2.364277 \\ 41 & 8 & 0 & 4.645196 & -0.933127 & -1.984893 \\ 42 & 1 & 0 & 4.160540 & -1.763107 & -1.843320 \\ 43 & 1 & 0 & -4.108469 & -0.827430 & -2.431214 \\ 44 & 8 & 0 & -4.437154 & -1.628193 & -1.990128 \\ 45 & 1 & 0 & -5.185706 & -1.955303 & -2.504060 \\ ------------------------------------------------------------------\end{array}$

\section{$\mathrm{UO}_{2}\left(\mathrm{H}_{2} \mathrm{O}\right)_{4}\left(\mathrm{H}_{2} \mathrm{O}\right)_{11}{ }^{2+} \mathrm{C}_{1}$ (B3LYP)}

Standard orientation:

\begin{tabular}{|c|c|c|c|c|c|}
\hline \multirow{2}{*}{$\begin{array}{l}\text { Center } \\
\text { Number }\end{array}$} & \multirow{2}{*}{\multicolumn{2}{|c|}{$\begin{array}{l}\text { Atomic } \\
\text { Number }\end{array}$}} & \multirow{2}{*}{$\begin{array}{l}\text { Atomic } \\
\text { Type }\end{array}$} & \multicolumn{2}{|c|}{ Coordinates (Angstroms } \\
\hline & & & & $\mathrm{X}$ & $\mathrm{Z}$ \\
\hline 1 & 92 & 0 & -0.159127 & -0.225292 & 0.008862 \\
\hline 2 & 8 & 0 & 0.318231 & -1.168864 & 1.425459 \\
\hline 3 & 8 & 0 & -0.650953 & 0.734391 & -1.395462 \\
\hline 4 & 8 & 0 & -2.089626 & -1.530070 & -0.208778 \\
\hline 5 & 1 & 0 & -2.384154 & -2.242590 & 0.418923 \\
\hline 6 & 1 & 0 & -2.878202 & -1.308800 & -0.816752 \\
\hline 7 & 1 & 0 & -1.957433 & 2.031378 & 0.927284 \\
\hline 8 & 8 & 0 & -1.421864 & 1.315190 & 1.338846 \\
\hline 9 & 1 & 0 & -1.611857 & 1.291653 & 2.315678 \\
\hline 10 & 8 & 0 & 1.774358 & 1.079125 & 0.239531 \\
\hline 11 & 1 & 0 & 2.009990 & 1.881540 & -0.330030 \\
\hline 12 & 1 & 0 & 2.562130 & 0.816270 & 0.792905 \\
\hline 13 & 1 & 0 & 1.754424 & -2.355990 & -0.990291 \\
\hline 14 & 8 & 0 & 1.103370 & -1.687543 & -1.351999 \\
\hline 15 & 1 & 0 & 1.226794 & -1.627539 & -2.336522 \\
\hline 16 & 1 & 0 & 3.566884 & -3.113752 & 0.255705 \\
\hline 17 & 8 & 0 & 2.906013 & -3.357571 & -0.419942 \\
\hline 18 & 1 & 0 & 2.869107 & -4.320566 & -0.463071 \\
\hline 19 & 1 & 0 & 0.792034 & -1.772708 & -4.652934 \\
\hline 20 & 8 & 0 & 1.452969 & -1.506499 & -4.000642 \\
\hline 21 & 1 & 0 & 2.313580 & -1.608154 & -4.427585 \\
\hline
\end{tabular}




$\begin{array}{rrrrrr}22 & 1 & 0 & 4.235635 & -0.602238 & 1.647824 \\ 23 & 8 & 0 & 3.885119 & 0.307875 & 1.636696 \\ 24 & 1 & 0 & 4.257024 & 0.773396 & 2.395185 \\ 25 & 1 & 0 & 2.312902 & 3.094185 & -2.179776 \\ 26 & 8 & 0 & 2.262048 & 3.164710 & -1.219024 \\ 27 & 1 & 0 & 2.881276 & 3.884241 & -0.953077 \\ 28 & 8 & 0 & -2.008490 & 1.330297 & 3.927887 \\ 29 & 1 & 0 & -1.506678 & 1.765802 & 4.628822 \\ 30 & 1 & 0 & -2.633048 & 0.734256 & 4.360995 \\ 31 & 8 & 0 & -2.922123 & 3.242792 & -0.012597 \\ 32 & 1 & 0 & -3.151480 & 4.058972 & 0.456010 \\ 33 & 1 & 0 & -2.458689 & 3.523087 & -0.816107 \\ 34 & 1 & 0 & -3.956323 & -3.621455 & 1.429939 \\ 35 & 8 & 0 & -3.025050 & -3.372719 & 1.501417 \\ 36 & 1 & 0 & -2.559576 & -4.153241 & 1.828532 \\ 37 & 8 & 0 & -4.159192 & -0.957070 & -1.589194 \\ 38 & 1 & 0 & -4.556948 & -0.068194 & -1.423667 \\ 39 & 1 & 0 & -4.317310 & -1.184289 & -2.513035 \\ 40 & 1 & 0 & 5.749458 & -2.468737 & 1.135512 \\ 41 & 8 & 0 & 4.849464 & -2.421635 & 1.491333 \\ 42 & 1 & 0 & 4.879470 & -2.895792 & 2.335433 \\ 43 & 1 & 0 & -4.516965 & 2.196372 & -0.646181 \\ 44 & 8 & 0 & -5.087711 & 1.509981 & -1.027886 \\ 45 & 1 & 0 & -6.000693 & 1.816673 & -0.974798 \\ 46 & 1 & 0 & 3.673437 & 6.099342 & -0.394255 \\ 47 & 8 & 0 & 3.954970 & 5.180307 & -0.487746 \\ 48 & 1 & 0 & 4.912671 & 5.202134 & -0.609169 \\ --------------------------------------------------------------\end{array}$

\section{$\mathrm{UO}_{2}\left(\mathrm{H}_{2} \mathrm{O}\right)_{5}\left(\mathrm{H}_{2} \mathrm{O}\right)_{7}{ }^{2+} \mathrm{C}_{1}(\mathrm{~B} 3 \mathrm{LYP})$}

Standard orientation:

\begin{tabular}{|c|c|c|c|c|c|}
\hline \multirow{2}{*}{$\begin{array}{l}\text { Center } \\
\text { Number }\end{array}$} & \multirow{2}{*}{$\begin{array}{l}\text { Atomic } \\
\text { Number }\end{array}$} & \multirow{2}{*}{\multicolumn{2}{|c|}{$\begin{array}{l}\text { Atomic } \\
\text { Type }\end{array}$}} & \multicolumn{2}{|c|}{ Coordinates (Angstron } \\
\hline & & & & $\mathrm{X}$ & $\mathrm{Z}$ \\
\hline 1 & 92 & 0 & 0.008638 & -0.049453 & 0.001691 \\
\hline 2 & 8 & 0 & 0.025177 & -0.080814 & -1.761025 \\
\hline 3 & 8 & 0 & -0.010638 & 0.040747 & 1.762361 \\
\hline 4 & 8 & 0 & -1.702913 & 1.692842 & 0.010161 \\
\hline 5 & 1 & 0 & -2.306540 & 1.937938 & -0.740109 \\
\hline 6 & 1 & 0 & -1.558271 & 2.484569 & 0.564571 \\
\hline 7 & 1 & 0 & -2.735596 & -1.161155 & 0.894683 \\
\hline 8 & 8 & 0 & -2.141910 & -1.204258 & 0.097538 \\
\hline 9 & 1 & 0 & -2.341943 & -2.034226 & -0.378529 \\
\hline 10 & 8 & 0 & 2.383034 & -0.601658 & 0.120447 \\
\hline 11 & 1 & 0 & 2.927947 & -0.462438 & 0.941353 \\
\hline 12 & 1 & 0 & 2.800389 & -1.321285 & -0.393129 \\
\hline 13 & 1 & 0 & 1.739190 & 2.477124 & -0.706804 \\
\hline 14 & 8 & 0 & 1.190452 & 2.087901 & 0.024592 \\
\hline
\end{tabular}




$\begin{array}{rrrrrr}15 & 1 & 0 & 0.841743 & 2.814453 & 0.576975 \\ 16 & 1 & 0 & -0.374215 & -3.150351 & -0.494043 \\ 17 & 8 & 0 & 0.345888 & -2.566163 & -0.183298 \\ 18 & 1 & 0 & 1.189764 & -2.918198 & -0.530490 \\ 19 & 1 & 0 & 3.561414 & -3.670645 & -0.707065 \\ 20 & 8 & 0 & 2.968796 & -3.046771 & -1.152340 \\ 21 & 1 & 0 & 3.157126 & -3.135552 & -2.098274 \\ 22 & 1 & 0 & -2.226512 & -3.933566 & -2.011674 \\ 23 & 8 & 0 & -2.058726 & -3.769949 & -1.071864 \\ 24 & 1 & 0 & -2.458844 & -4.515474 & -0.599925 \\ 25 & 8 & 0 & -3.349983 & 2.431996 & -1.956225 \\ 26 & 1 & 0 & -4.298815 & 2.583167 & -1.856568 \\ 27 & 1 & 0 & -3.140872 & 2.587560 & -2.886056 \\ 28 & 8 & 0 & -3.774172 & -1.160717 & 2.204482 \\ 29 & 1 & 0 & -3.556324 & -1.404401 & 3.113308 \\ 30 & 1 & 0 & -4.715828 & -0.946204 & 2.191144 \\ 31 & 1 & 0 & 2.429416 & 3.345307 & -2.824397 \\ 32 & 8 & 0 & 2.672110 & 3.188017 & -1.903038 \\ 33 & 1 & 0 & 3.557928 & 3.554096 & -1.783121 \\ 34 & 1 & 0 & -0.520079 & 3.708230 & 2.507832 \\ 35 & 8 & 0 & -0.522557 & 3.774312 & 1.541451 \\ 36 & 1 & 0 & -0.653029 & 4.714016 & 1.345922 \\ 37 & 1 & 0 & 3.689559 & -0.642704 & 3.183227 \\ 38 & 8 & 0 & 3.905808 & -0.344647 & 2.290296 \\ 39 & 1 & 0 & 4.754731 & 0.112696 & 2.347306 \\ & 1 & & & \\ - & & & & & \\ 36 & & & \end{array}$

\section{$\mathrm{UO}_{2}\left(\mathrm{H}_{2} \mathrm{O}\right)_{5}\left(\mathrm{H}_{2} \mathrm{O}\right)_{10}{ }^{2+} \mathrm{C}_{1}$ (B3LYP)}

Standard orientation:

\begin{tabular}{cccccc}
\hline Center & \multicolumn{2}{c}{ Atomic } & \multicolumn{2}{c}{ Atomic } & \multicolumn{2}{c}{ Coordinates } \\
Number & Number & Type & X & Y & $Z$ \\
--------- & $Z$ \\
1 & 92 & 0 & 0.130438 & -0.005265 & -0.016073 \\
2 & 8 & 0 & 0.185506 & 0.069225 & 1.747093 \\
3 & 8 & 0 & 0.157230 & -0.081599 & -1.781871 \\
4 & 8 & 0 & 2.086097 & 1.440508 & -0.178926 \\
5 & 1 & 0 & 2.714396 & 1.513651 & 0.594085 \\
6 & 1 & 0 & 2.623788 & 1.508749 & -0.993849 \\
7 & 1 & 0 & 2.749065 & -1.497420 & -0.618459 \\
8 & 8 & 0 & 2.093687 & -1.439264 & 0.131919 \\
9 & 1 & 0 & 2.599449 & -1.512436 & 0.967151 \\
10 & 8 & 0 & -2.394372 & -0.012405 & -0.002919 \\
11 & 1 & 0 & -2.966571 & -0.806986 & -0.070378 \\
12 & 1 & 0 & -2.972449 & 0.772676 & 0.114808 \\
13 & 1 & 0 & -1.069379 & 2.730341 & 0.793052 \\
14 & 8 & 0 & -0.742733 & 2.261687 & -0.027084 \\
15 & 1 & 0 & -1.130247 & 2.749765 & -0.778121 \\
16 & 1 & 0 & -0.992334 & -2.794812 & -0.753214
\end{tabular}




$\begin{array}{rrrrrr}17 & 8 & 0 & -0.728642 & -2.275174 & 0.031143 \\ 18 & 1 & 0 & -1.175518 & -2.713178 & 0.808774 \\ 19 & 1 & 0 & -2.281561 & -3.780704 & 2.688292 \\ 20 & 8 & 0 & -2.259478 & -3.512368 & 1.761779 \\ 21 & 1 & 0 & -3.148737 & -3.220460 & 1.501007 \\ 22 & 1 & 0 & -2.147951 & -3.769318 & -2.822553 \\ 23 & 8 & 0 & -2.180316 & -3.849719 & -1.858243 \\ 24 & 1 & 0 & -2.129534 & -4.799446 & -1.673511 \\ 25 & 1 & 0 & 4.001596 & -0.503705 & -2.212356 \\ 26 & 8 & 0 & 3.893115 & -1.375362 & -1.790982 \\ 27 & 1 & 0 & 4.212416 & -2.049232 & -2.403136 \\ 28 & 1 & 0 & 3.271947 & -1.644180 & 3.344885 \\ 29 & 8 & 0 & 3.683916 & -1.374533 & 2.511070 \\ 30 & 1 & 0 & 4.520834 & -1.860352 & 2.464931 \\ 31 & 8 & 0 & 3.822504 & 1.403094 & 1.800380 \\ 32 & 1 & 0 & 3.883634 & 0.537366 & 2.243458 \\ 33 & 1 & 0 & 4.073770 & 2.083798 & 2.436584 \\ 34 & 8 & 0 & 3.850123 & 1.412315 & -2.442753 \\ 35 & 1 & 0 & 3.544257 & 1.721135 & -3.307974 \\ 36 & 1 & 0 & 4.684049 & 1.877938 & -2.280260 \\ 37 & 1 & 0 & -1.836036 & 3.805659 & 2.796085 \\ 38 & 8 & 0 & -1.990996 & 3.557017 & 1.876436 \\ 39 & 1 & 0 & -2.895142 & 3.209351 & 1.798652 \\ 40 & 1 & 0 & -2.684427 & 3.876699 & -2.560392 \\ 41 & 8 & 0 & -2.524543 & 3.871621 & -1.605598 \\ 42 & 1 & 0 & -2.429032 & 4.801820 & -1.351863 \\ 43 & 1 & 0 & -3.722306 & 2.899450 & -0.450613 \\ 44 & 8 & 0 & -3.972754 & 2.301143 & 0.281303 \\ 45 & 1 & 0 & -4.938687 & 2.286289 & 0.315332 \\ 46 & 1 & 0 & -3.572283 & -2.924920 & -0.880884 \\ 47 & 8 & 0 & -3.962159 & -2.354775 & -0.189012 \\ 48 & 1 & 0 & -4.916766 & -2.344449 & -0.340722 \\ ----------------------------------------------------------------\end{array}$

